\title{
THE CHOW MOTIVES OF RELATIVE FULTON-MACPHERSON SPACE
}

\author{
FUMITOSHI SATO
}

\begin{abstract}
Suppose that $X$ is a complex nonsingular projective variety and $D$ is a smooth divisor. Compactifications of configuration spaces of distinct and non-distinct $n$ points in $X$ away from $D$ were constructed by the author and B. Kim in "A generalization of Fulton-MacPherson configuration spaces" by using the method of wonderful compactification. In this paper, we give explicit presentations of Chow motives and Chow groups of these configuration spaces.
\end{abstract}

\section{Introduction}

Let $X$ be a complex connected nonsingular projective algebraic variety and let $D$ be a smooth divisor. In [4], two generalizations of Fulton-MacPherson spaces were constructed by using the method of wonderful compactification [5]. These spaces were important because they were used to give simple constructions of moduli of relative stable maps and logarithmic stable maps [1], [3].

Two spaces are defined as following:

(1) A compactification $X_{D}^{[n]}$ of the configuration space of $n$ labeled points in $X \backslash D$, i.e. "not allowing those points to meets $D$."

(2) A compactification $X_{D}[n]$ of the configuration space of $n$ distinct labeled points in $X \backslash D$, i.e. "not allowing those points to meet each other as well as $D$.'

The goal of this paper is to give an explicit presentation of Chow motives and Chow groups of these configuration spaces. Our main theorems are:

THEOREM 1.1. We have the Chow group and motive decompositions

$$
\begin{aligned}
A^{*}\left(X_{D}^{[n]}\right) & =\bigoplus_{\mathscr{C} \mathscr{H}} \bigoplus_{\vec{\mu} \in M_{\mathscr{G} \mathscr{C}}} A^{*-\|\vec{\mu}\|}\left(D_{S_{\mathscr{G} \mathscr{C}}}\right), \\
h\left(X_{D}^{[n]}\right) & =\bigoplus_{\mathscr{C} \mathscr{H}} \bigoplus_{\vec{\mu} \in M_{\mathscr{H} \mathscr{C}}} h\left(D_{S_{\mathscr{G C}}}\right)(\|\vec{\mu}\|),
\end{aligned}
$$

Received 3 August 2011, in final form 3 February 2012. 
where $\mathscr{C} \mathscr{H}$ runs through all the chains of $\{1,2, \ldots, n\}, S_{\mathscr{C} \mathscr{H}}$ is the maximal element in $\mathscr{C} \mathscr{H}$ and $\|\cdot\|$ is the $l_{1}$ norm.

THEOREM 1.2. We have the Chow group and motive decompositions

$$
\begin{aligned}
A^{*}\left(X_{D}[n]\right) & =\bigoplus_{\mathcal{N}} \bigoplus_{\vec{\mu} \in M_{\mathcal{N}}}\left(\bigoplus_{\mathscr{C} \mathscr{C}} \bigoplus_{\vec{\lambda} \in M_{\mathscr{C} \mathscr{C}}} A^{*-\|\vec{\mu}\|-\|\vec{\lambda}\|}\left(D_{S_{\mathscr{C} \mathscr{C}}}\right)\right), \\
h\left(X_{D}[n]\right) & =\bigoplus_{\mathcal{N}} \bigoplus_{\vec{\mu} \in M_{\mathcal{H}}}\left(\bigoplus_{\mathscr{C} \mathscr{H}} \bigoplus_{\vec{\lambda} \in M_{\mathscr{C} \mathscr{C}}} h\left(D_{S_{\mathscr{C} \mathscr{C}}}\right)(\|\vec{\mu}\|+\|\vec{\lambda}\|)\right),
\end{aligned}
$$

where $\mathcal{N}$ runs through all the nests of $\{1,2, \ldots, n\}$ and $\mathscr{C} \mathscr{H}$ runs through all the chains whose length is the number of connected components of the forest which corresponds to $\mathcal{N}$.

The paper is organized as follows. In section 2, we review theory of wonderful compactification and Chow motives after blow-up. In section 3, we review the construction of compactifications of $n$ points in $X \backslash D$. In section 4 , we compute Chow groups and motives explicitly.

\subsection{Notation}

- As in [2], for a subset $I$ of $N:=\{1,2, \ldots, n\}$, let

$$
I^{+}:=I \cup\{n+1\} .
$$

- Let $\mathrm{Bl}_{Z} Y$ be the blow-up of a nonsingular complex projective variety $Y$ along a nonsingular closed subvariety $Z$.

- Let $Y_{1}$ be the blow-up of a nonsingular complex projective variety $Y_{0}$ along a nonsingular closed subvariety $Z$. If $V$ is an irreducible subvariety of $Y_{0}$, we will use $\widetilde{V}$ or $V\left(Y_{1}\right)$ to denote

- the total transform of $V$, if $V \subseteq Z$;

- the proper transform of $V$, otherwise.

If there is no risk to cause confusion, we will use simply $V$ to denote $\widetilde{V}$. The space $\mathrm{Bl}_{\widetilde{V}} Y_{1}$ will be called the iterated blow-ups of $Y_{0}$ along centers $Z, V$ (with the order). When we want to indicate where an iterated transform of $V$ lives explicitly, we will write it $V\left(Y_{n}\right)$.

- For a partition of $I$ of $N, \Delta_{I}$ denotes the polydiagonal associated to $I$. And consider a binary operation $I \wedge J$ on the set of all partitions satisfying

$$
\Delta_{I} \cap \Delta_{J}=\Delta_{I \wedge J}
$$

We use $\Delta_{I_{0}}$ instead of $\Delta_{I}$ when $I=\left\{I_{0}, I_{1}, \ldots, I_{l}\right\}$ such that $\left|I_{i}\right|=1$ for all $i \geq 1$. 


\subsection{Acknowledgements}

We would like to thank B. Kim and L. Li for many valuable discussions. Most part of this work was done during our visit at Mittag-Leffler Institute. We thanks for its hospitality. This research was supported by Japan Society for the Promotion of Science, Grant 21840056003.

\section{Wonderful Compactification of Arrangements of Subvarieties}

In this section, we review the theory of wonderful compactification of arrangements of subvarieties. See the detail and proofs in [5], [6].

\subsection{Arrangement, building set and nest}

Definition 2.1 (Of clean intersection). Let $Y$ be a complex nonsingular projective algebraic variety and let $U$ and $V$ be two smooth subvarieties of $Y$. We say that $U$ and $V$ intersect cleanly if $U \neq V$ and their scheme-theoretic intersection is nonsingular and the tangent bundles satisfy $T(U \cap V)=T U \cap$ $T V$.

REMARK 2.2. If the intersection is transversal, then it is a clean intersection.

Definition 2.3 (Of simple arrangement). A simple arrangement of subvarieties of $Y$ is a finite set $\mathscr{S}=\left\{S_{i}\right\}$ of nonsingular closed irreducible subvarieties of $Y$ satisfying the following conditions

(1) $S_{i}$ and $S_{j}$ intersect cleanly,

(2) $S_{i} \cap S_{j}$ is either empty or some $S_{k}$ 's.

Definition 2.4 (Of building set). Let $\mathscr{S}$ be a simple arrangement of subvarieties of $Y$. A subset $\mathscr{G} \subseteq \mathscr{S}$ is called a building set with respect to $\mathscr{S}$, if , for any $S \in \mathscr{S}$, the minimal elements in $\mathscr{G}$ which contain $S$ intersect transversally and their intersection is $S$. These minimal elements are called the $\mathscr{G}$-factors of $S$.

Definition 2.5 (Of $\mathscr{G}$-nest). A subset $\mathscr{T} \subseteq \mathscr{G}$ is called a $\mathscr{G}$-nest if there is a flag of elements in $\mathscr{S}: S_{1} \subset S_{2} \subset \cdots \subset S_{k}$ such that

$$
\mathscr{T}=\bigcup_{i=1}^{k}\left\{A: A \text { is a } \mathscr{G} \text {-factor of } S_{i}\right\} .
$$




\subsection{Construction of $Y_{\mathscr{G}}$ by a sequence of blow-ups}

Let $Y$ be a complex nonsingular projective algebraic variety, $\mathscr{S}$ be a simple arrangement of subvarieties of $Y$ and $\mathscr{G}$ be a building set with respect to $\mathscr{S}$. Or$\operatorname{der} \mathscr{G}=\left\{G_{1}, \ldots, G_{N}\right\}$ such that $i<j$ if $G_{i} \subset G_{j}$. We define $\left(Y_{k}, \mathscr{S}^{(k)}, \mathscr{G}^{(k)}\right)$ inductively, where $Y_{k}$ is a blow-up of $Y_{k-1}$ along a nonsingular variety, $\mathscr{S}^{(k)}$ is a simple arrangement of subvarieties of $Y_{k}$ and $\mathscr{G}^{(k)}$ is a building set with respect to $\mathscr{S}^{(k)}$.

THEOREM 2.6. Assume $\mathscr{S}$ is a simple arrangement of subvarieties of $Y$ and $\mathscr{G}$ is a building set. Let $G$ be a minimal element in $\mathscr{G}$ and consider $\pi: \widetilde{Y}:=$ $\mathrm{Bl}_{G} Y \rightarrow Y$. Denote the exceptional divisor by $E$. For any nonsingular variety $V$ in $Y$, we define $\widetilde{V} \subset \mathrm{Bl}_{G} Y$, the $\sim$-transform of $V$, to be the proper transform of $V$ if $V \nsubseteq G$, and to be $\pi^{-1}(V)$ if $V \subseteq G$.

For simplicity of notation, for a sequence of blow-ups, we use the same notation $\widetilde{V}$ to denote the iterated one.

(1) The collection $\mathscr{S}^{\prime}$ of subvarieties in $\widetilde{Y}$ defined by

$$
\mathscr{S}^{\prime}:=\{\tilde{S}\}_{S \in \mathscr{S}} \cup\{\tilde{S} \cap E\}_{\emptyset \subset S \cap G \subset S}
$$

is a simple arrangement in $\tilde{Y}$

(2) $\mathscr{G}^{\prime}:=\left\{\widetilde{G_{i}}\right\}_{G_{i} \in \mathscr{G}}$ is a building set with respect to $\mathscr{S}^{\prime}$.

(3) Given a subset $\mathscr{T}$ of $\mathscr{G}$, define $\mathscr{T}^{\prime}:=\{\tilde{A}\}_{A \in \mathscr{T}} \subseteq \mathscr{G}^{\prime} . \mathscr{T}$ is a $\mathscr{G}$-nest if and only if $\mathscr{T}^{\prime}$ is a $\mathscr{G}^{\prime}$-nest.

Let's go back to the construction of $Y_{\mathscr{G}}$.

(1) For $k=0, Y_{0}=Y, \mathscr{S}^{(0)}=\mathscr{S}, \mathscr{G}^{(0)}=\mathscr{G}=\left\{G_{1}, \ldots, G_{N}\right\}, G_{i}^{(0)}=G_{i}$.

(2) Assume $Y_{k-1}$ is already constructed. Let $Y_{k}$ be the blow-up of $Y_{k-1}$ along the nonsingular subvariety $G_{k}^{(k-1)}$. Define $G_{i}^{(k)}:=G_{i}^{(k-1)}$. Since $G_{i}^{(k-1)}$ for $i<k$ are all divisors, $G_{k}^{(k-1)}$ is minimal in $\mathscr{G}^{(k-1)}$. Thus there is a naturally induced simple arrangement $\mathscr{S}^{(k)}$ and a building set $\mathscr{G}^{(k)}$ by the Theorem 2.6.

(3) Continue the inductive construction to $k=N$, where all elements in the building set $\mathscr{G}^{(N)}$ are divisors.

TheOREM 2.7. Denote $Y^{\circ}=Y \backslash \cup_{G \in G} G$. There is a natural locally closed embedding

$$
Y^{\circ} \hookrightarrow Y \times \prod_{G \in \mathscr{G}} \mathrm{Bl}_{G} Y,
$$

and its cloure is denoted by $Y_{G}$ and called the wonderful compactification of $Y$ with respect to $\mathscr{G}$. Then $Y_{\mathscr{G}}$ is isomorphic to $Y_{N}$ which is constructed in the 
above. The variety $Y_{\mathscr{G}}$ is nonsingular. For each $G \in \mathscr{G}$, there is a nonsingular divisors $D_{G} \subset Y_{\mathscr{G}}$ such that

(1) The union of these divisors is $Y_{\mathscr{G}} \backslash Y^{\circ}$.

(2) Any set of these divisors meets transversally. An intersection of divisors $D_{T_{1}} \cap \cdots \cap D_{T_{l}}$ is not empty exactly when $\left\{T_{1}, \ldots, T_{l}\right\}$ forms a $\mathscr{G}_{\text {-nest. }}$

THEOREM 2.8 (Order of blow-ups).

(1) Let $\mathscr{I}_{i}$ be the ideal sheaf of $G_{i} \in \mathscr{G}$. Then

$$
Y_{\mathscr{G}} \cong \mathrm{B} 1_{\mathscr{I}_{1} \ldots \mathscr{I}_{N}} Y
$$

(2) If we arrange $\mathscr{G}=\left\{G_{1}, \ldots, G_{N}\right\}$ in such an order that

(*) for any $1 \leq i \leq N$, the first $i$ terms $G_{1}, \ldots, G_{i}$ form a building set.

Then

$$
Y_{\mathscr{G}} \cong \mathrm{Bl}_{\widetilde{G_{N}}} \ldots \mathrm{Bl}_{\widetilde{G_{2}}} \mathrm{Bl}_{G_{1}} Y,
$$

where each blow-up is along a smooth subvariety.

\subsection{Chow group and motive of $Y_{G}$}

Let $Y_{0}:=Y, Y_{0} \mathscr{T}:=\cap_{T \in \mathscr{T}} T$ where $\mathscr{T}$ is a $\mathscr{G}_{\text {-nest. Define }} r_{\mathscr{T}}(G):=$ $\operatorname{dim}\left(\cap_{G \subset T \in \mathscr{T} T}\right)-\operatorname{dim} G$ (here we use a convention that $\cap_{G \subset T \in \mathscr{T}} T=Y$ if no $T$ strictly contains $G$ ). Then define

$$
M_{\mathscr{T}}:=\left\{\vec{\mu}=\left\{\mu_{G}\right\}_{G \in \mathscr{T}}: 1 \leq \mu_{G} \leq r_{\mathscr{T}}(G)-1\right\} .
$$

Let $\|\vec{\mu}\|:=\sum_{G \in \mathscr{G}} \mu_{G}$ for $\vec{\mu} \in M_{\mathscr{T}}$.

THEOREM 2.9. We have the Chow group decomposition

$$
A^{*}\left(Y_{\mathscr{G}}\right)=A^{*}(Y) \oplus \bigoplus_{\mathscr{T}} \bigoplus_{\vec{\mu} \in M_{\mathscr{T}}} A^{*-\|\vec{\mu}\|}\left(Y_{0} \mathscr{T}\right)
$$

where $\mathscr{T}$ runs through all $\mathscr{G}$-nests. We also have the Chow motive decomposition

$$
h\left(Y_{\mathscr{G}}\right)=h(Y) \oplus \bigoplus_{\mathscr{T}} \bigoplus_{\vec{\mu} \in M_{\mathscr{T}}} h\left(Y_{0} \mathscr{T}\right)(\|\vec{\mu}\|)
$$

where $\mathscr{T}$ runs through all $\mathscr{G}$-nests. 


\section{Construction of $X_{D}^{[n]}$ and $X_{D}[n]$}

Fix a nonsingular divisor $D$ of a complex nonsingular projective algebraic variety $X$ of dimension $m$. In this section, we review constructions of a compactification of configuration space of $n$ points in $X \backslash D, X_{D}^{[n]}$, and a compactification of configuration space of $n$ distinct points in $X \backslash D, X_{D}[n]$. In this paper, we assume that $D$ is a divisor but every thing will work in the case where $D$ is a smooth subvariety after some adjustment. See the details in [4].

\subsection{Construction}

For a subset $S$ of $N:=\{1,2, \ldots, n\}$ define a nonsingular subvariety in $X^{n}$

$$
D_{S}:=\left\{\mathbf{x} \in X^{n} \mid \mathbf{x}_{i} \in D, \forall i \in S\right\} .
$$

Let $\mathscr{A}$ be the collection of $D_{S}$ for all $S \subseteq N:=\{1, \ldots, n\}$ with $|S| \geq 2$. It is clear that the collection is a simple arrangement of smooth subvarieties of $X^{n}$. Take a building set $\mathscr{G}=\mathscr{A}$. Then define $X_{D}^{[n]}$ to be the closure of $X^{n} \backslash \bigcup_{S} D_{S}$ in

$$
X^{n} \times \prod_{S} \mathrm{Bl}_{D_{S}} X^{n}
$$

It can be constructed by a successive blow-ups by Theorem 2.7. In particular we may order $\mathscr{G}$ as $D_{12} ; D_{123} ; D_{13}, D_{23} ; \ldots ; D_{12 \ldots . .} ; D_{U \cup\{n\}}$ with $|U|=n-2$ and $U \subset N \backslash\{n\} ; \ldots ; D_{\text {in }}$ for $i=1, \ldots, n-1$ by Theorem 2.8 .

For $I \subseteq N$ with $|I| \geq 2,\left\{\Delta_{I}\left(X_{D}^{[n]}\right)\right\}$ forms a building set of nonsingular subvarieties of $X_{D}^{[n]}$ with respect to the set of $\sim$-transforms of all polydiagonals. So we define $X_{D}[n]$ as followings. in

Definition 3.1. Define $X_{D}[n]$ to be the closure of $X_{D}^{[n]} \backslash \bigcup_{|I| \geq 2} \Delta_{I}\left(X_{D}^{[n]}\right)$

$$
X_{D}^{[n]} \times \prod_{|I| \geq 2} \mathrm{Bl}_{\Delta_{I}\left(X_{D}^{[n]}\right)} X_{D}^{[n]}
$$

Then, it satisfies the following properties.

THEOREM 3.2.

(1) $X_{D}[n]$ is a nonsingular variety. There is a natural projection from $X_{D}[n]$ to $X_{D}[|I|]$ for any subset I of $N$. There is a natural $S_{n}$-action on $X_{D}[n]$.

(2) The boundary is the union of divisors $\widetilde{D_{S}}$ with $|S| \geq 1$, and $\widetilde{\Delta_{I}}$ with $|I| \geq 2$ of normal crossings.

(3) The intersections of boundary divisors are nonempty if and only if they are nested. Here $\left\{D_{S_{i}}, \Delta_{I_{j}}\right\}$ is nested if each pair $S_{i}$ and $S_{i^{\prime}}\left(I_{j}\right.$ and $\left.I_{j^{\prime}}\right)$ is either disjoint or one is contained in the other and each pair $S_{i}$ and $I_{j}$ is either disjoint or $I_{j}$ is contained in $S_{i}$. 
(4) We may take a following order of blow-ups: $D_{S}$; $\Delta_{I}$ for $n \notin S, I ; D_{T}$ with $n \in T ; \Delta_{J}$ with $n \in J$.

This is a summary of Theorem 1 and 2 in [4].

\section{Chow groups and motives}

In this section, we will apply Theorem 2.9 to $X_{D}^{[n]}$ and $X_{D}[n]$.

\subsection{Chow group and motive of $X_{D}^{[n]}$}

In this case, our $Y=X^{n}, \mathscr{S}=\mathscr{G}=\left\{D_{S}: S \subseteq N\right.$ with $\left.|S| \geq 2\right\}$ where $D_{S}=\left\{\mathbf{x} \in X^{n} \mid \mathbf{x}_{i} \in D, \forall i \in S\right\}$. We have $\mathscr{S}=\mathscr{G}$, so a $\mathscr{G}$-nest $\mathscr{T}$ is just a chain of elements in $\mathscr{S}$, that is $\mathscr{T}=\left\{D_{S_{1}} \subset D_{S_{2}} \subset \cdots \subset D_{S_{k}}\right\}$. Thus $Y_{0} \mathscr{T}=D_{S_{1}}$.

A chain $\mathscr{C} \mathscr{H}$ is a chain of proper subset of $N, S_{k} \subset \cdots \subset S_{2} \subset S_{1}$, such that $S_{k}$ is not a singleton. Obviously, there is one-to-one correspondence between a set of chains of $\mathscr{S}$ and a set of chains of $N$. We say $\emptyset$ is also a chain. We define $\max _{\mathscr{C} \mathscr{H}(\mathscr{T})} S$ as the maximal element of $\mathscr{C} \mathscr{H}(\mathscr{T})$ which is strictly contained in $S$, where $\mathscr{C} \mathscr{H}(\mathscr{T})$ is the chain of $N$ which corresponds to $\mathscr{T}$. If there is no such element, then we define $\max _{\mathscr{C} \mathscr{H}(\mathscr{T})} S=\emptyset$

Now let $G=D_{S}$ and let's compute $r_{\mathscr{T}}(G)$;

$$
\begin{aligned}
r_{\mathscr{T}}(G) & =\operatorname{dim}\left(\bigcap_{G \subset T \in \mathscr{T}} T\right)-\operatorname{dim} G \\
& =\operatorname{dim}\left(D_{\max _{\mathscr{G} \mathscr{C}}(\mathcal{T})} S\right)-\operatorname{dim} D_{S} \\
& =|S|-\left|\max _{\mathscr{C} \mathscr{H}(\mathscr{T})} S\right| .
\end{aligned}
$$

Remark 4.1 (When $D$ is not a divisor). When $D$ is not a divisor, then we also blow up along $D_{\{i\}}$. So we will not exclude the case such that $S_{k}$ is a singleton for $\left\{S_{k} \subset \cdots \subset S_{2} \subset S_{1}\right\}$. The definition of $r_{\mathscr{T}}(G)$ will be also changed. It will be multiplied by the codimension of $D$ in $X$. See more details in [6].

For a chain $\mathscr{C} \mathscr{H}(\neq \emptyset)$, define

$$
M_{\mathscr{C} \mathscr{H}}:=\left\{\vec{\mu}=\left\{\mu_{S}\right\}_{S \in \mathscr{C} \mathscr{H}}: 1 \leq \mu_{S} \leq|S|-\left|\max _{\mathscr{C} \mathscr{H}} S\right|-1\right\} .
$$

For $\mathscr{C} \mathscr{H}=\emptyset$, define $M_{\mathscr{C} \mathscr{C}}$ to be the set consisting of one $\vec{\mu}$ with $\|\vec{\mu}\|=0$ and $D_{\emptyset}=X^{n}$. 
THEOREM 4.2 (Theorem 1.1). We have the Chow group and motive decompositions

$$
\begin{aligned}
A^{*}\left(X_{D}^{[n]}\right) & =\bigoplus_{\mathscr{C} \mathscr{H}} \bigoplus_{\vec{\mu} \in M_{\mathscr{G} \mathscr{C}}} A^{*-\|\vec{\mu}\|}\left(D_{S_{\mathscr{G} \mathscr{C}}}\right), \\
h\left(X_{D}^{[n]}\right) & =\bigoplus_{\mathscr{C} \mathscr{H}} \bigoplus_{\vec{\mu} \in M_{\mathscr{G} \mathscr{C}}} h\left(D_{S_{\mathscr{C} \mathscr{C}}}\right)(\|\vec{\mu}\|),
\end{aligned}
$$

where $\mathscr{C} \mathscr{H}$ runs through all the chains of $N$ and $S_{\mathscr{C} \mathscr{H}}$ is the maximal element in $\mathscr{C} \mathscr{H}$.

\subsection{Chow group and motive of $X_{D}[n]$}

We use the same notation as [6].

(1) We call two subsets $I, J \subseteq N$ are overlapped if $I \cap J$ is not a nonempty proper subset of both $I$ and $J$. For a set $\mathscr{N}$ of subsets of $N$, we call $I$ is compatible with $\mathcal{N}$, denoted by $I \sim \mathcal{N}$, if $I$ does not overlap any elements of $\mathcal{N}$. A nest $\mathscr{N}$ is a set of subset of $N$ such that any pair $I \neq J \in \mathscr{N}$ are not overlapped and contains all singletons. For a given nest $\mathcal{N}$, define $\mathscr{N}^{\circ}:=\mathscr{N} \backslash\{\{1\}, \ldots,\{n\}\}$. A nest $\mathcal{N}$ naturally corresponds to a tree (which may not be connected) with each node being labeled by an element of $\mathcal{N}$. Let $c(\mathcal{N})$ be the number of connected components of the forest which corresponds to $\mathscr{N}$. Denote by $c_{I}(\mathcal{N})$ the number of maximal elements of the set $\{J \in \mathscr{N}: J \subset I\}$, which is called the number of sons of the node $I$. Let $\overline{\Delta_{\mathcal{N}}}:=\cap_{I \in \mathcal{N}} \Delta_{I}\left(X_{D}^{[n]}\right)$ in this section.

(2) For a nest $\mathcal{N}(\neq\{\{1\}, \ldots,\{n\}\})$, define

$$
M_{\mathcal{N}}:=\left\{\vec{\mu}=\left\{\mu_{I}\right\}_{I \in \mathcal{N}}: 1 \leq \mu_{I} \leq m\left(c_{I}(\mathcal{N})-1\right)-1\right\}
$$

where $m=\operatorname{dim} X$. For $\mathcal{N}=\{\{1\}, \ldots,\{n\}\}$, define $M_{\mathcal{N}}=\{\vec{\mu}\}$ with $\|\vec{\mu}\|=0$.

As in [6], we have

Proposition 4.3. We have the Chow group and motive decompositions

$$
\begin{aligned}
A^{*}\left(X_{D}[n]\right) & =\bigoplus_{\mathcal{N}} \bigoplus_{\vec{\mu} \in M_{\mathcal{N}}} A^{*-\|\vec{\mu}\|}\left(\overline{\Delta_{\mathcal{N}}}\right), \\
h\left(X_{D}[n]\right) & =\bigoplus_{\mathcal{N}} \bigoplus_{\vec{\mu} \in M_{\mathcal{N}}} h\left(\overline{\Delta_{\mathcal{N}}}\right)(\|\vec{\mu}\|),
\end{aligned}
$$

where $\mathcal{N}$ runs through all the nests of $N$ 
Now we need to simplify $A^{*}\left(\overline{\Delta_{\mathscr{N}}}\right)$ and $h\left(\overline{\Delta_{\mathscr{N}}}\right)$.

Lemma 4.4. $D_{S}$ and $\Delta_{I}$ intersect cleanly.

Proof. We only need to prove that $T D_{S} \cap T \Delta_{I} \subset T\left(D_{S} \cap \Delta_{I}\right)$. An arc in $\Delta_{I}$ have a coordinate representative $\left(\mathbf{x}_{i}\right) \in X^{n}$ such that $\mathbf{x}_{i}=\mathbf{x}_{j}$ for $i, j \in I$. For an arc in $\Delta_{I}$ to be an arc in $D_{S}, \mathbf{x}_{i} \in D$ for all $i \in S$. Thus the arc should be an $\operatorname{arc}$ in $D_{S} \cap \Delta_{I}$.

Proposition 4.5. $\overline{\Delta_{I}}$ is isomorphic to $X_{D}^{\left[\left.\right|^{c} \mid+1\right]}$.

Proof. We need to know which blow-ups along $D_{S}$ have an effect to $\Delta_{I}$ in a specific order of blow-ups. We can assume that $I=\{l, \cdots, n\}$ by arranging the order. Then denote $a=\left|I^{c}\right|$ and $b=|I|$. We will denote $\Delta_{I}$ by $X^{a} \times \Delta$ $\left(\cong X^{\left|I^{c}\right|+1}\right)$. Then we have two different kinds of $D_{S}$. The first one is that $S \subset I^{c}$, which we call the first kind, the second one is that $S \nsubseteq I^{c}$, which we call the second kind. We will change the order of blow-ups so that we first blow up along $D_{S}$ 's of the first kind, and then along the second kind. More precisely, we order $D_{I^{c}} \times X^{b}, D_{1, \ldots, \hat{i}, \ldots, l} \times X^{b}, \ldots, D_{i, j} \times X^{b}(i, j \in$ $\{1, \ldots, a\})$ and then $D_{I^{c}} \times D^{b}, \ldots, D_{S^{\prime}} \times D_{S^{\prime \prime}}, \ldots\left(\left|S^{\prime \prime}\right|>0\right.$ and $\left(\left|S^{\prime}\right|,\left|S^{\prime \prime}\right|\right)$ : non-increasing in lexicographical order). This order satisfies $(*)$-condition in Theorem 2.6, so that we can blow up in this order. In this order of blow-ups, notice that $X^{a} \times \Delta$ and $D_{S^{\prime}} \widetilde{\times} D_{S^{\prime \prime}}$ for $S^{\prime \prime} \subset I$ are separated when we blow up along $D_{S^{\prime}} \times D^{b}$. Thus we can forget the process of blow-ups by $D_{S^{\prime}} \widetilde{\times} D_{S^{\prime \prime}}$ where $S^{\prime \prime} \subset I$ i.e. we only need to care about $D_{S^{\prime}} \times D^{b}$ for the second kind. Under the isomorphism $X^{a} \times \Delta \cong X^{a+1}$, they are just $D_{S^{\prime}} \times D$.

We can also apply the same technique to polydiagonals term by term. Thus we can go further from proposition 4.3.

THEOREM 4.6 (Theorem 1.2). We have the Chow group and motive decompositions

$$
\begin{aligned}
A^{*}\left(X_{D}[n]\right) & =\bigoplus_{\mathcal{N}} \bigoplus_{\vec{\mu} \in M_{\mathcal{N}}}\left(\bigoplus_{\mathscr{C} \mathscr{H}} \bigoplus_{\vec{\lambda} \in M_{\mathscr{G} \mathscr{C}}} A^{*-\|\vec{\mu}\|-\|\vec{\lambda}\|}\left(D_{S_{\mathscr{G} \mathscr{C}}}\right)\right), \\
h\left(X_{D}[n]\right) & =\bigoplus_{\mathcal{N}} \bigoplus_{\vec{\mu} \in M_{\mathcal{H}}}\left(\bigoplus_{\mathscr{C H}} \bigoplus_{\vec{\lambda} \in M_{\mathscr{G}}} h\left(D_{S_{\mathscr{H}}}\right)(\|\vec{\mu}\|+\|\vec{\lambda}\|)\right),
\end{aligned}
$$

where $\mathcal{N}$ runs through all the nests of $N$ and $\mathscr{C} \mathscr{H}$ runs through all the chains whose length is $c(\mathcal{N})$. 


\section{REFERENCES}

1. Abramovich, D., and Fantechi, B., Orbifold techniques in degeneration formulas, arXiv: 1103.5132.

2. Fulton, W., and MacPherson, R., A compactification of configuration spaces, Ann. of Math. 139 (1994), 183-225.

3. Kim, B., Logarithmic stable maps, pp. 167-200 in: New developments in algebraic geometry, integrable systems and mirror symetry, Adv. Stud. Pure Math. 59, Math. Soc. Japan, Tokyo 2010.

4. Kim, B., and Sato, F., A generalization of Fulton-MacPherson configuration spaces, Selecta Math. (N.S.) 15 (2009), 435-443.

5. Li, L., Wonderful compactifications of arrangements of subvarieties, Michigan Math. J. 58 (2009), 535-563.

6. Li, L., Chow motive of Fulton-MacPherson configuration spaces and wonderful compactifications, Michigan Math. J. 58 (2009), 565-598.

KAGAWA NATIONAL COLLEGE OF TECHNOLOGY

355 CHOKUSHI-CHO, TAKAMATSU

KAGAWA 761-8058

JAPAN

E-mail: fumi@math.utah.edu 